\title{
Upper gastrointestinal involvement in systemic sclerosis: Findings in a real-life setting
}

\author{
ANA PETCU ${ }^{1}$, LINDA JESSICA GHIB ${ }^{1}$, SIMONA MIHAELA GRAD ${ }^{2}$, CORNELIA POPOVICI $^{3}$, \\ LILIANA ROGOJAN $^{4}$, NICOLAE VOICU REDNIC ${ }^{5}$ and SIMONA REDNIC $^{1}$ \\ ${ }^{1}$ Rheumatology Department and ${ }^{2}$ 2nd Medical Clinic, 'Iuliu Haţieganu' University of Medicine and Pharmacy; \\ ${ }^{3}$ 1st Medical Clinic, Gastroenterology Department and ${ }^{4}$ Department of Pathology, \\ Emergency Clinical County Hospital, 400006 Cluj-Napoca; ${ }^{5}$ rd Medical Clinic, Gastroenterology Department, \\ 'Iuliu Haţieganu' University of Medicine and Pharmacy, 400162 Cluj-Napoca, Romania
}

Received August 30, 2019; Accepted October 1, 2019

DOI: $10.3892 /$ etm.2019.8125

\begin{abstract}
Gastrointestinal tract involvement is the most common visceral affectation in systemic sclerosis (SSc), but the manifestations may vary in extension and severity. Endoscopic and histopathological gastroesophageal findings were investigated in patients with SSc. A total of 79 consecutive patients with definite SSc were enrolled in a cross sectional study. Clinical data were collected, upper gastrointestinal endoscopy and biopsies from gastric mucosa were performed in all cases. Fifty-seven (72.1\%) out of $79 \mathrm{SSc}$ patients had gastroesophageal symptoms. The most frequent were dysphagia, present in $33(41.7 \%)$ and gastroesophageal reflux symptoms in $23(29.1 \%)$ patients. Out of the 79 patients, 22 were asymptomatic, but in 16 esophageal and gastric mucosa changes were endoscopically detected. Reflux esophagitis was found in 39 (49.3\%) patients. The presence of esophageal manifestations was not related to the disease duration or with its other variables. Signs of gastritis were endoscopically described in 47 (59.4\%) and confirmed on histopathologic examinations in 45 patients. In 31 patients without any endoscopic changes, 18 (22.7\%) showed signs of gastritis on histopathologic examination. No significant statistical differences were found between symptomatic and asymptomatic patients or between those with limited cutaneous SSc and those with diffuse cutaneous SSc in terms of clinical, endoscopic or histopathological findings, except the higher proportion of hiatal hernia in symptomatic patients. The results of this study might suggest that upper gastrointestinal endoscopy should be performed during the early stage
\end{abstract}

Correspondence to: Ms. Ana Petcu, Rheumatology Department, 'Iuliu Haţieganu' University of Medicine and Pharmacy, 2-4 Clinicilor Street, 400006 Cluj-Napoca, Romania

E-mail: ana03mai@yahoo.com

Key words: systemic sclerosis, gastroesophageal manifestations, upper gastrointestinal endoscopy, histopathological findings, gastritis of the disease and then periodically in patients diagnosed with SSc, even in the absence of typical symptoms.

\section{Introduction}

Systemic sclerosis (SSc) is a rare rheumatic disease in which vasculopathy, tissue fibrosis and autoimmunity are pathophysiological features that lead to specific forms of organ damage. According to LeRoy criteria patients with SSc can present with skin thickening proximal to the elbow and knee in diffuse cutaneous SSc (dcSSc) or with skin thickening restricted to areas distal to the elbow and knee in limited cutaneous SSc (lcSSc) (1). Involvement of the gastrointestinal tract (GIT) is one of the common systemic manifestations of SSc, besides skin findings and Raynaud's phenomenon (2). Gastroesophageal manifestations, present both in lcSSc and dcSSc, may significantly affect the quality of life and in rare cases they can cause lethal complications (3). Data from a Central-Eastern Europe cohort support the hypothesis that the GIT involvement is more frequent than expected (3). Moreover, in a multicenter collaboration analysis of 7,655 patients, on the large European League Against Rheumatism Scleroderma Trials and Research (EUSTAR) cohort, upper GIT manifestations were more frequently described, as compared to lower GIT symptoms (4). Both the esophagus and the stomach can be involved resulting in a variety of symptoms from dysphagia and regurgitation to retrosternal burning pain, early satiety and epigastric pain (2).

As clinical symptoms are non-specific and the diagnosis is often delayed, upper gastrointestinal (GI) endoscopy is used to highlight the substrate of these symptoms, which usually is esophagitis or gastritis. If left untreated, they may lead to further complications such as ulceration on the esophagus and the stomach, Barrett's esophagus, benign esophageal strictures and adenocarcinoma, which also can be diagnosed by endoscopy $(5,6)$. Upper GI endoscopy is also useful in diagnosing gastric antral vascular ectasia (GAVE) and candidiasis in SSc patients $(6,7)$. GAVE or 'watermelon stomach' has a unique endoscopic appearance, with prominent multiple red vessels in a stripe pattern, radiating from the antrum to the pylorus (8). 
The main goal of the present study was to describe gastroesophageal endoscopic and histopathological findings in patients with SSc irrespective of the presence of gastroesophageal symptoms. Differences between dcSSc and lcSSc in terms of upper GIT involvement were compared.

\section{Materials and methods}

Study population. In this cross-sectional study, 79 consecutive patients with SSc attending the inpatient clinic of the Rheumatology Department of the Emergency Clinical County Hospital (Cluj-Napoca, Romania) between November 2016 and December 2018 were enrolled. All patients met the ACR/EULAR 2013 classification criteria for SSc (9). We assessed esophagitis and gastritis from a clinical, endoscopic and histopathological point of view.

Study approval was obtained from the institutional ethics review board and all subjects signed a written informed consent according to the Declaration of Helsinki.

Exclusion criteria were: i) severe cardiopulmonary involvement (i.e., restrictive lung disease); ii) diabetes, iii) bisphosphonate use; iv) active smokers; v) patients with current alcohol consumption; and vi) patients with markedly reduced oral aperture, who were unable to secure mouth guard during the endoscopic procedure.

Data collection. Data were collected regarding demographic and clinical features, including information on age, gender, disease duration (defined as the onset of the first non-Raynaud's symptom), type of skin involvement, presence or absence of gastroesophageal symptoms (heartburn, acid regurgitation, dysphagia, early satiety, epigastric pain), the presence of cardiovascular, pulmonary and renal manifestations. The presence of digital ulcers, calcinosis, telangiectasias, arthritis, anemia, dyslipidemia and current or previous medication intake were also collected from all subjects.

Routine laboratory studies included C-reactive protein (CRP) levels. Antinuclear, anticentromere and anti-topoisomerase-I antibodies were measured.

Upper gastrointestinal endoscopy. All SSc patients, irrespective of the presence or absence of gastroesophageal symptoms, underwent upper GI endoscopy for detection of esophageal and gastric mucosa lesions. Endoscopy was performed after an overnight fast using an Olympus videogastroscope EVIS EXERA III (GIF-HQ190).

Mucosal damage of the esophagus was classified according to the Los Angeles (LA) Classification (9). The diagnosis of esophageal candidiasis was made by endoscopy and was defined by the presence of white or slightly yellowish plaquelike lesions on the esophageal mucosa as described in a previous study (10). Barrett's esophagus was defined by the presence of a specialized columnar epithelium with a minimum length of $1 \mathrm{~cm}$ (tongues or circular) into the distal esophagus and the presence of specialized intestinal metaplasia on histopathological examination in accordance to recent European Society of Gastrointestinal Endoscopy guidelines (11). In addition, the presence or absence of a hiatal hernia was noted.

An abnormal-appearing gastric mucosa (edema, erythema, friability, erosions, fold enlargement, fold atrophy, visibility of the vascular pattern, intramural bleeding, nodularity) was defined as gastritis on endoscopy (12). To establish a definitive diagnosis of gastritis, minimum four biopsies were taken from the gastric mucosal tissue ( 2 from the antrum and 2 from corpus) using a standard biopsy forceps. Additional biopsies were taken from any area of abnormality.

The histopathological examination assessed the presence of polymorphonuclear leucocytes alongside the mononuclear inflammatory infiltrate, intestinal metaplasia, atrophy characterized by the loss of the normal mucosal glands. The presence of Helicobacter pylori (HP) organisms was also assessed on histopathological examination.

Statistical analysis. Statistical analysis was performed with SPSS version 21 (SPSS Inc., Chicago, IL, USA). P<0.05 was considered to indicate a statistically significant difference. Results are expressed as the mean (standard deviation) for continuous numerical data, median (25.75 percentiles) for discrete numerical data or frequency (percentage) for categorical variables. The normality distribution test was performed with the aid of the Shapiro-Wilk test. Comparisons across groups were performed with the Student's t-test for normally distributed data and with Mann-Whitney U test for those that were not following normal distribution. The Chi-square test was used to compare frequencies among the populations.

\section{Results}

A total of 79 eligible SSc patients were enrolled: 7 males (8.8\%) and 72 females $(91.1 \%)$. The mean age was $53.4 \pm 9.8$ years (range, 23-77). The median disease duration at the time of the study was 6.01 years. A total of $44(55.6 \%)$ patients had lcSSc and 35 (44.3\%) had dcSSc. A majority of these patients (94.9\%) received a disease-modifying antirheumatic drugs (DMARDs). Among DMARDs, methotrexate was the most frequently prescribed $(46.8 \%)$, followed by azathioprine $(43.1 \%)$ and cyclophosphamide (29.1), in association with corticosteroids (44\%) and non-steroidal anti-inflammatory drugs (NSAIDS) (32.9\%). There was no association between drug intake and gastroesophageal symptoms or endoscopic findings (data not shown). Demographics, clinical data and medication use in the two subsets of patients are shown in Table I.

The most frequent upper gastrointestinal manifestations were dysphagia, present in 16 (36.4\%) lcSSc and $17(48.6 \%)$ dcSSc patients, and gastroesophageal reflux in $10(22.7 \%)$ lcSSc and 13 (37.1\%) dcSSc patients. Endoscopic examination revealed that esophagitis (LA classification A-D) was present in $19(43.1 \%)$ of lcSSc patients and $20(57.1 \%)$ of those with dcSSc. Twelve out of $26(52.1 \%)$ patients with gastroesophageal reflux symptoms had esophagitis on endoscopy. There were no statistically significant differences between patients with lcSSc and patients with dcSSc in terms of gastroesophageal symptoms, endoscopic findings and esophagitis grading (Table II). Barrett's esophagus was diagnosed by endoscopy, according to predefined criteria in 8 patients, but only 2 of those with dcSSc and 3 of those with lcSSc were histopathologically confirmed. In one case the endoscopy could not evaluate the stomach due to the presence of esophageal stenosis. Forty-five $(56.9 \%)$ patients had gastric abnormalities on endoscopic 
Table I. Patient and disease characteristics.

\begin{tabular}{|c|c|c|c|}
\hline Characteristics & $\begin{array}{c}\mathrm{lcSSc} \\
\mathrm{n}=44(\%)\end{array}$ & $\begin{array}{c}\mathrm{dcSSc} \\
\mathrm{n}=35(\%)\end{array}$ & P-value \\
\hline Age & $55.1(10.6)$ & $51.29(11.3)$ & 0.1 \\
\hline Female & $43(97.7)$ & $29(82.9)$ & 0.04 \\
\hline Disease duration (years) & $6.73(5)$ & $5.1(4.2)$ & 0.1 \\
\hline ANA positivity & $43(97.7)$ & $34(97.1)$ & 0.9 \\
\hline \multicolumn{4}{|l|}{ Capillaroscopy pattern } \\
\hline Normal & $1(2.3)$ & $1(2.8)$ & 0.5 \\
\hline Early & $17(38.6)$ & $9(25.7)$ & \\
\hline Active & $18(40.9)$ & $18(51.4)$ & \\
\hline Late & $8(18.2)$ & $6(17.1)$ & \\
\hline Rodnan score & $4(2 ; 8)$ & $12.5(6 ; 17)$ & $<0.001$ \\
\hline 6MWT n $(\%)$ & $372(109)$ & $344(125)$ & 0.1 \\
\hline $\begin{array}{l}\text { Presence of } \\
\text { telangiectasia }\end{array}$ & $32(72.7)$ & $19(54.3)$ & 0.08 \\
\hline NSAID use & $14(31.8)$ & $12(34.3)$ & 0.8 \\
\hline Arthralgia & $32(72.7)$ & $27(77.1)$ & 0.4 \\
\hline Arthritis & $9(20.5)$ & $8(22.9)$ & 0.5 \\
\hline PPI use prior to UE & $34(77.3)$ & $30(85.7)$ & 0.3 \\
\hline Bosentan/Sildenafil & $7(15.9)$ & $4(11.4)$ & 0.5 \\
\hline
\end{tabular}

Numerical data are expressed as mean (SD) and categorical data as no. (\%). 1cSSc, limited cutaneous systemic sclerosis; dcSSc, diffuse cutaneous systemic sclerosis; ANA, antinuclear antibodies; 6MMWT, 6 minutes walking test; NSAID, non steroidal antiinflammatory drug; PPI, proton pump inhibitor; UE, upper endoscopy.

evaluation and were confirmed by histopathological examinations. Thirteen (16.4\%) patients were positive for HP on direct histopathological visualization. Thirty-one $(39.2 \%)$ patients had no signs of gastritis on endoscopy, but histopathological signs of gastritis such as inflammatory plasma cells, lymphocytes, neutrophil infiltration and intestinal metaplasia were present in $18(22.7 \%)$ of them. There were no significant statistical differences between those with $\mathrm{lcSSc}$ and respectively those with dcSSc in terms of endoscopic or histopathological gastritis $(\mathrm{P}=0.2$ and 0.4 , respectively, in the Chi-Square test). Endoscopic appearance of watermelon stomach was found in 2 patients and of esophageal candidiasis in 4. Sixty-five out of 79 patients were treated with proton pump inhibitors (PPI) (Omeprazole $20 \mathrm{mg} /$ day, mean duration of administration was 8.9 months). Thirty-seven out of these 65 patients $(56.9 \%)$ had esophagitis and $46(70.7 \%)$ had gastritis. There were no significant statistical differences between clinical or endoscopic findings and age, disease duration or other disease variables.

In total, 22 patients (27.8\%) were asymptomatic concerning gastroesophageal manifestations, but in $72.7 \%$ endoscopic changes were found. There were no statistically significant differences between symptomatic and asymptomatic patients in terms of endoscopic and histopathological findings (Table III), except the significantly higher presence of hiatal hernia in symptomatic patients $(\mathrm{P}=0.01)$.
Table II. Main clinical and endoscopic findings in patients with lcSSc compared to patients with dcSSc.

\begin{tabular}{lccc}
\hline Characteristics & $\begin{array}{c}\text { lcSSc } \\
\mathrm{n}=44(\%)\end{array}$ & $\begin{array}{c}\mathrm{dcSSc} \\
\mathrm{n}=35(\%)\end{array}$ & P-value \\
\hline GE symptoms & $32(72.7)$ & $25(71.4)$ & 0.8 \\
Dysphagia & $16(36.4)$ & $17(48.6)$ & 0.3 \\
GE reflux & $10(22.7)$ & $13(37.1)$ & 0.3 \\
Nausea/Vomiting & $2(4.5)$ & $4(11.4)$ & 0.3 \\
Early satiety & $3(6.8)$ & $5(14.3)$ & 0.4 \\
Epigastric pain & $7(15.9)$ & $3(8.6)$ & 0.5 \\
Hiatal hernia & $7(15.9)$ & $12(34.2)$ & 0.06 \\
Esophagitis & & & \\
Class A & $7(15.9)$ & $11(31.4)$ & 0.2 \\
Class B & $8(18.2)$ & $4(11.4)$ & \\
Class C & $3(6.8)$ & $2(5.7)$ & \\
Class D & $1(2.3)$ & $3(8.6)$ & \\
Barrett esophagus & $4(9.1)$ & $4(11.4)$ & 0.7 \\
Endoscopic gastritis & $29(65.9)$ & $19(54.2)$ & 0.8 \\
Histopathologic gastritis & $37(84.1)$ & $27(77.1)$ & 0.7 \\
HP positive & $7(15.9)$ & $5(14.3)$ & 0.6 \\
Esophageal ulcer & $4(9.1)$ & $1(2.9)$ & 0.2 \\
Intestinal metaplasia & $4(9.1)$ & $3(8.6)$ & 0.9 \\
\hline
\end{tabular}

Data are expressed as no. (\%). lcSSc, limited cutaneous systemic sclerosis; dcSSc, diffuse cutaneous systemic sclerosis; GE, gastroesophageal; HP, Helicobacter pylori.

Table III. Comparison of symptomatic and asymptomatic patients in terms of endoscopic and histopathological findings.

\begin{tabular}{lccc}
\hline Values & $\begin{array}{c}\text { Symptomatic } \\
\mathrm{n}=57(\%)\end{array}$ & $\begin{array}{c}\text { Asymptomatic } \\
\mathrm{n}=22(\%)\end{array}$ & P-value \\
\hline Hiatal hernia & $18(31.6)$ & $1(4.5)$ & 0.01 \\
Gastric polyps & $6(10.5)$ & $1(4.5)$ & 0.4 \\
Metaplasia & $5(8.8)$ & $2(9.1)$ & 0.9 \\
Esophageal ulcers & $4(7)$ & $1(4.5)$ & 0.6 \\
HP positivity & $8(14)$ & $5(2.7)$ & 0.3 \\
Endoscopic gastritis & $43(75.4)$ & $13(59.1)$ & 0.2 \\
Histopathologic & $46(80)$ & $18(81)$ & 0.9 \\
gastritis & & & \\
Barrett esophagus & $7(12.3)$ & $1(4.5)$ & 0.3 \\
Esophagitis & $31(54.4)$ & $8(36.4)$ & 0.2 \\
\hline
\end{tabular}

Data are expressed as n (\%). HP, Helicobacter pylori.

\section{Discussion}

The purpose of this study was to assess gastroesophageal involvement in a group of 79 consecutive SSc patients using 
upper GI endoscopy. Possible differences were evaluated between the subtypes of the disease, regardless of the presence or absence of symptoms. Several publications assessing GI damage in patients with SSc suggest a high prevalence of gastroesophageal involvement (estimated up to 90\%) (13). Some studies highlighted the fact that SSc patients showed damage in the upper GI tract even in the absence of symptoms and early in the disease (14-16). In our study, gastroesophageal symptoms were present in $72.1 \%$ of cases, both in early and late stages of the disease. A large proportion of asymptomatic patients $(72.7 \%)$ experienced esophageal and gastric changes in endoscopy.

The average age of disease onset for SSc in our study was 47.3 years (similar to the EUSTAR update report) (4). The disease duration of the majority of our patients (64.5\%) was under 6 years. Dysphagia was present in $33(41.7 \%)$ patients and gastroesophageal reflux symptoms in 23 (29.1\%) patients, while both symptoms were present in 13 cases (16.4\%), similar when compared with other studies (17-19). The relationship between esophageal symptoms and the subtype of SSc is controversial. Lahcene et al (17) found that esophageal complaints were significantly more frequent in dcSSc, while in studies of Bassotti et al (20) and Akesson and Wollheim (13), as well as in our work, they were not related with disease subtype.

In total, 19 patients $(24.05 \%)$ were found with hiatal hernia on endoscopy, with significantly higher frequency of hiatal hernia in symptomatic patients (94\%) and in the diffuse disease subtype (63.1\%). Similar results were observed in other studies in patients with SSc $(3,21,22)$. Also, association between hiatal hernia and reflux symptoms, reflux esophagitis, Barrett's esophagus and esophageal adenocarcinoma was observed in several studies in the general population (23-25).

In our study, reflux esophagitis was found in 40 cases (50.6\% of the patients), similar with the prevalence reported in several previous recent studies $(17,19)$. Complications of reflux esophagitis such as esophageal stenosis, Barrett esophagus and esophageal adenocarcinoma are increased in SSc (26). In our study, Barrett esophagus has been endoscopically described in $8(10.1 \%)$ cases but only 5 cases have been histopathologically confirmed. The prevalence of Barrett's esophagus reported in some retrospective studies varies between 2 and 37\% (26-28). Data on the effects of PPI treatment on esophageal and gastric mucosal inflammation in SSc patients are heterogeneous. PPI treatment with maximal doses (lansoprazole $2 \times 30 \mathrm{mg}$ or pantoprazole $2 \times 40 \mathrm{mg}$ ) is useful for long-term safety and reduces the length of Barrett esophagus (29). Some other reports suggested that patients with SSc should be encouraged to receive early and long-term PPI treatment $(14,19)$. Thonhofer et al $(14)$ showed in a small group of patients with early SSc who received adequate PPI therapy and antibiotic therapy for elimination of HP, complete mucosal healing in the distal esophagus and stomach (14). On the contrary, Hendel et al (6) reported that complete healing of esophagitis occurs only in half of the patients with SSc and severe gastroesophageal reflux disease receiving intensive PPI treatment. The results of our study showed that, although a large proportion of patients were under PPI treatment at the time of endoscopy, most of them had esophagitis and/or gastritis. This could be explained by the administration of low doses of PPI and the use of concomitant medication (such as NSAIDS, DMARDs, Bosentan). There was one case of esophageal stenosis and no case of adenocarcinoma observed in our study.

Although there have been several publications addressing the frequency and intensity of gastric symptoms in SSc patients, the majority focused on the evaluation of delayed gastric emptying, gastroparesis and the presence of GAVE. In our study, the focus was on the presence of gastritis in SSc patients, which was encountered at endoscopy in 47 (59.4\%) and histopathologically confirmed in 45 patients. In 18 patients gastritis was detected only after histopathological examination. In a Central-Eastern Europe cohort, gastritis was present in $18.3 \%$ of SSc patients (3). In a retrospective study by Thonhofer et al (14), 12 out of 13 patients with early SSc without GE symptoms had endoscopic gastritis. This wide range of prevalence in diagnosis of gastritis could be due to differences between diagnosis protocols and different PPI usage in the above studies.

HP is commonly associated with various autoimmune diseases, but its role in inducing them is controversial $(30,31)$. Some data suggest that HP infection is correlated with SSc activity and skin involvement (32). In our patients, we did not find any differences between dcSSc and lcSSc regarding the presence of HP. Despite the fact that in the last decades there has been a decline in the prevalence of chronic gastritis and HP infection, their frequency in East European countries remain high $68.9 \%$ in Romania and 66.6\% in Poland for HP prevalence) (33). The prevalence of HP infection in our study was $15.1 \%$, much lower than we expected $(31,32)$, probably due to continuous PPI treatment.

Our study highlights the fact that SSc patients with or without gastroesophageal symptoms should be endoscopically evaluated, given that patients experience esophageal and gastric disease irrespective of disease duration or the presence of PPI treatment. We still need more studies to evaluate when and how often endoscopy should we performed.

The main limitation of our study is the relatively small sample size and the fact that endoscopy was performed at different stages of the disease. Also, our cohort was heterogeneous in terms of disease duration and disease-related complications and many of our patients were undergoing PPI, DMARDS or NSAIDS treatment which could have influenced the results.

Our data support the results of other authors, according to which SSc patients often have gastroesophageal involvement during the early phase of the disease and in the absence of symptoms. Our data also suggest that PPI treatment can be the cause of the lack of symptoms in those with endoscopic gastroesophageal damage.

In conclusion, upper GI involvement was frequent in SSc patients, with no significant differences between dcSSc and lcSSc. Endoscopic changes were present in the majority of the asymptomatic patients. Our findings suggest the need of upper GI endoscopy in the management of SSc patients.

\section{Acknowledgements}

Not applicable. 


\section{Funding}

No funding was received.

\section{Availability of data and materials}

The datasets used and/or analyzed during the current study are available from the corresponding author on reasonable request.

\section{Authors' contributions}

AP and LJG designed the study. AP, LJG and NVR collected the clinical data. AP, LJG and NVR were responsible for the data analysis. AP, LJG and SR contributed to data interpretation and writing the manuscript. SMG and CP performed the endoscopies. LR was responsible for the histopathological examinations. All the authors approved the final manuscript.

\section{Ethics approval and consent to participate}

The study was approved by the Ethics Committee of the 'Iuliu Haţieganu' University of Medicine and Pharmacy, (Cluj-Napoca, Romania) (no. 928/27.11.2013). Signed informed consents were obtained from the patients.

\section{Patient consent for publication}

Not applicable.

\section{Competing interests}

Authors declare that they have no competing interests.

\section{References}

1. LeRoy EC, Black C, Fleischmajer R, Jablonska S, Krieg T, Medsger TAJ Jr, Rowell N and Wollheim F: Scleroderma (systemic sclerosis): Classification, subsets and pathogenesis. J Rheumatol 15: 202-205, 1988.

2. Folwaczny C, Voderholzer W, Riepl RL and Schindlbeck N: Clinical aspects, pathophysiology, diagnosis and therapy of gastrointestinal manifestations of progressive systemic scleroderma. Z Gastroenterol 34: 497-508, 1996 (In German).

3. Szamosi S, Szekanecz Z and Szúcs G: Gastrointestinal manifestations in Hungarian scleroderma patients. Rheumatol Int 26: 1120-1124, 2006.

4. Meier FMP, Frommer KW, Dinser R, Walker UA, Czirjak L, Denton CP, Allanore Y, Distler O, Riemekasten G, Valentini G, et al; EUSTAR Co-authors: Update on the profile of the EUSTAR cohort: An analysis of the EULAR Scleroderma Trials and Research group database. Ann Rheum Dis 71: 1355-1360, 2012

5. Conio M, Blanchi S, Lapertosa G, Ferraris R, Sablich R, Marchi S, D'Onofrio V, Lacchin T, Iaquinto G, Missale G, et al: Long-term endoscopic surveillance of patients with Barrett's esophagus. Incidence of dysplasia and adenocarcinoma: A prospective study. Am J Gastroenterol 98: 1931-1939, 2003.

6. Hendel L, Svejgaard E, Walsøe I, Kieffer M and Stenderup A: Esophageal candidosis in progressive systemic sclerosis: Occurrence, significance, and treatment with fluconazole. Scand J Gastroenterol 23: 1182-1186, 1988.

7. Watson M, Hally RJ, McCue PA, Varga J and Jiménez SA: Gastric antral vascular ectasia (watermelon stomach) in patients with systemic sclerosis. Arthritis Rheum 39: 341-346, 1996.

8. Marie I, Ducrotte P, Antonietti M, Herve S and Levesque H: Watermelon stomach in systemic sclerosis: Its incidence and management. Aliment Pharmacol Ther 28: 412-421, 2008.
9. Armstrong D, Bennett JR, Blum AL, Dent J, De Dombal FT, Galmiche JP, Lundell L, Margulies M, Richter JE, Spechler SJ, et al: The endoscopic assessment of esophagitis: A progress report on observer agreement. Gastroenterology 111: 85-92, 1996.

10. Pech O: Esophageal Candidiasis. Video J Encycl GI Endosc 1: 64-65, 2013.

11. Weusten B, Bisschops R, Coron E, Dinis-Ribeiro M, Dumonceau JM, Esteban JM, Hassan C, Pech O, Repici A, Bergman J, et al: Endoscopic management of Barrett's esophagus: European Society of Gastrointestinal Endoscopy (ESGE) Position Statement. Endoscopy 49: 191-198, 2017.

12. Kaminishi M, Yamaguchi H, Nomura S, Oohara T, Sakai S, Fukutomi H, Nakahara A, Kashimura H, Oda M, Kitahora T, et al: Endoscopic classification of chronic gastritis based on the results of a pilot study by research society for gastritis. Gastroenterol Endosc 45: 1277-1291, 2003.

13. Akesson A and Wollheim FA: Organ manifestations in 100 patients with progressive systemic sclerosis: A comparison between the CREST syndrome and diffuse scleroderma. Br J Rheumatol 28: 281-286, 1989.

14. Thonhofer R, Siegel C, Trummer M and Graninger W: Early endoscopy in systemic sclerosis without gastrointestinal symptoms. Rheumatol Int 32: 165-168, 2012.

15. Jaovisidha K, Csuka ME, Almagro UA and Soergel KH: Severe gastrointestinal involvement in systemic sclerosis: Report of five cases and review of the literature. Semin Arthritis Rheum 34: 689-702, 2005.

16. Steen VD and Medsger TAJ Jr: Severe organ involvement in systemic sclerosis with diffuse scleroderma. Arthritis Rheum 43: 2437-2444, 2000.

17. Lahcene M, Oumnia N, Matougui N, Boudjella M, Tebaibia A and Touchene B: Esophageal involvement in scleroderma: Clinical, endoscopic, and manometric features. ISRN Rheumatol 2011: 325826, 2011.

18. Wielosz E, Borys O,Zychowska I and Majdan M: Gastrointestinal involvement in patients with systemic sclerosis. Pol Arch Med Wewn 120: 132-136, 2010.

19. Marie I, Ducrotte P, Denis P, Hellot MF and Levesque H: Oesophageal mucosal involvement in patients with systemic sclerosis receiving proton pump inhibitor therapy. Aliment Pharmacol Ther 24: 1593-1601, 2006.

20. Bassotti G, Battaglia E, Debernardi V, Germani U, Quiriconi F, Dughera L, Buonafede G, Puiatti P, Morelli A, Spinozzi F, et al: Esophageal dysfunction in scleroderma: Relationship with disease subsets. Arthritis Rheum 40: 2252-2259, 1997.

21. Zuber-Jerger I, Müller A, Kullmann F, Gelbmann CM, Endlicher E, Müller-Ladner U and Fleck M: Gastrointestinal manifestation of systemic sclerosis - thickening of the upper gastrointestinal wall detected by endoscopic ultrasound is a valid sign. Rheumatology (Oxford) 49: 368-372, 2010.

22. Wielosz E, Borys O,Żychowska I and Majdan M: Gastrointestinal involvement in patients with systemic sclerosis. Pol Arch Med Wewn 120: 132-136, 2010.

23. Petersen H, Johannessen T, Sandvik AK, Kleveland PM, Brenna E, Waldum H and Dybdahl JD: Relationship between endoscopic hiatus hernia and gastroesophageal reflux symptoms. Scand J Gastroenterol 26: 921-926, 1991.

24. Cameron AJ: Barrett's esophagus: Prevalence and size of hiatal hernia. Am J Gastroenterol 94: 2054-2059, 1999.

25. Weston AP, Badr AS and Hassanein RS: Prospective multivariate analysis of clinical, endoscopic, and histological factors predictive of the development of Barrett's multifocal high-grade dysplasia or adenocarcinoma. Am J Gastroenterol 94: 3413-3419, 1999.

26. Wipff J, Coriat R, Masciocchi M, Caramaschi P, Derk CT, Hachulla E, Riccieri V, Mouthon L, Krasowska D, Ananyeva LP, et al: Outcomes of Barrett's oesophagus related to systemic sclerosis: a 3-year EULAR Scleroderma Trials and Research prospective follow-up study. Rheumatology 50: 1440-1444, 2011.

27. Marie I, Dominique S, Levesque H, Ducrotté P, Denis P, Hellot MF and Courtois H: Esophageal involvement and pulmonary manifestations in systemic sclerosis. Arthritis Rheum 45: 346-354, 2001.

28. Recht MP, Levine MS, Katzka DA, Reynolds JC and Saul SH: Barrett's esophagus in scleroderma: Increased prevalence and radiographic findings. Gastrointest Radiol 13: 1-5, 1988. 
29. Gashi Z, Bahtiri E, Gashi A and Sherifi F: Proton Pump Inhibitors Diminish Barrett's Esophagus Length: Our Experience. Open Access Maced J Med Sci 6: 1041-1045, 2018.

30. Amital H, Govoni M, Maya R, Meroni PL, Ori B, Shoenfeld Y, Tincani A, Trotta F, Sarzi-Puttini P and Atzeni F: Role of infectious agents in systemic rheumatic diseases. Clin Exp Rheumatol 26 (Suppl 48): S27-S32, 2008.

31. Radić M: Role of Helicobacter pylori infection in autoimmune systemic rheumatic diseases. World J Gastroenterol 20: 12839-12846, 2014.

32. Radić M, Kaliterna DM and Radić J: Helicobacter pylori infection and systemic sclerosis - is there a link? Joint Bone Spine 78: 337-340, 2011.
33. Hooi JKY, Lai WY, Ng WK, Suen MMY, Underwood FE, Tanyingoh D, Malfertheiner P, Graham DY, Wong VWS, Wu JCY, et al: Global prevalence of Helicobacter pylori infection: Systematic Review and Meta-Analysis. Gastroenterology 153: 420-429, 2017.

(i) (3) This work is licensed under a Creative Commons Attribution-NonCommercial-NoDerivatives 4.0 International (CC BY-NC-ND 4.0) License. 